\title{
A Novel Method for Joint Motion Sensing on a Wearable Computer
}

\author{
Aaron Toney, \\ Department of Electrical Engineering \\ University of Washington \\ Box 352-500 \\ Seattle, WA 98195, USA \\ joeboy@u.washington.edu
}

\begin{abstract}
Some wearable computing applications require sensing devices that detect the deflection of joints during human motion. Presented here is a novel non-invasive technique for measuring joint motion using pressure sensors. Described is a specific example of this; an easy to assemble glove that can be used as a low cost, high resolution gesture input device for wearable computers.
\end{abstract}

\section{1: Introduction}

In every day activity, the human body provides an infinite supply of information. To be effective, wearable computers require an interface that reacts to the gestures and motions of the person wearing the computer. In order to create such natural and organic interfaces, data about the force, position, and orientation of the user is needed by the computer. The pressure volume relations presented here can be used to construct the needed sensors for angular deflection, torsional stress, and contact pressure.

\section{2: Theoretical Background}

The Helmholtz equation gives the relationship between pressure $\mathbf{P}$, temperature $\mathbf{T}$, and gram molecular weight $\mathbf{n r}$, of gas molecules contained in a volume $\mathbf{V}$.

$$
\mathbf{P V}=\mathbf{n r T} \quad \text { Eqn. } 1
$$

For a sealed chamber, the total amount of gas, nr, will be constant. Furthermore, with active calibration by integrated temperature sensors[1], $\mathbf{T}$ has a manageable effect. Under these conditions, changes in volume proportionately change pressure.

\section{$P \propto 1 / V$}

Eqn. 2
The volume of a sealed elastic cylindrical tube will change as it is bent. A tube bent over a body joint, for example, will change in volume-and thus pressure-as it is articulated.

The sensitivity of the angular measure relies on generating the greatest proportional change in volume for a given angular displacement. The diameter of the cylinder determines both the total volume, and that volume's dependence on curvature. The smaller the volume the greater the sensitivity.

\section{3: The Data Glove}

To demonstrate the viability of using pressure sensors for a wearable interface, we constructed a data glove. Sealed air-filled tubes were mounted along the fingers and pressure sensing electronics placed on the back of the hand as shown in figure 1.
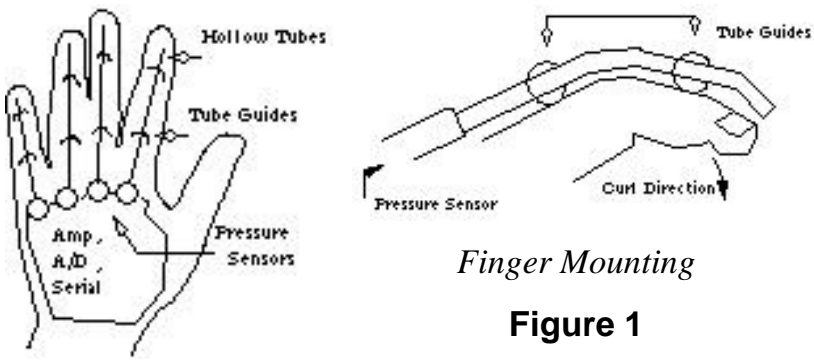

Finger Mounting

Figure 1

Figure 2 shows a layout of the angles derived for each finger in the glove. We assume the joints of the finger can move freely, and that the joints are not articulated independently of each other. Under these conditions, the first joint's bend angle $\boldsymbol{C}$ is in direct proportion to the second joint's angle $\boldsymbol{A}$. If we know the total length of the finger $\boldsymbol{L 1}+\boldsymbol{L} \mathbf{2}+\boldsymbol{L} \mathbf{3}$ and the length of segment $\boldsymbol{L} \boldsymbol{2}$, from an initial calibration of the system then we can measure the total curvature of the finger $\boldsymbol{B}$, and compute the bend of joints $\boldsymbol{A}$ and $\boldsymbol{C}$. So a single sensor 
can be used to measure the curvature of the finger.

\section{Figure 2}

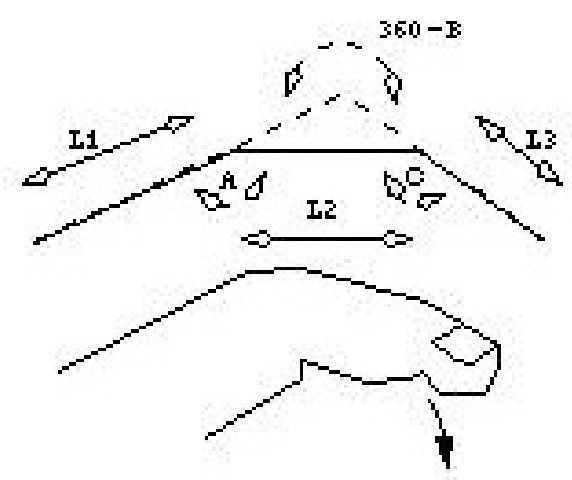

\section{3: Materials}

The tubes used were 2-mm internal diameter PVC, commonly used for fish tank air pumps. Four MPX2010 pressure sensors were used [2]. These each have a typical voltage swing of $20 \mathrm{mV}$, which was amplified to 2.5 Volts and noise filtered out using a simple LM351 OP amp with a low pass RC filter on its input. Data was sampled by a PIC 16c84, which controlled a D/A with a MUX and the serial interface. Pressure data is sampled from each finger at a rate of $240 \mathrm{~Hz}$ and transmitted back to the computer at $9600 \mathrm{bps}$. The total cost of the initial prototype was fortyeight dollars.

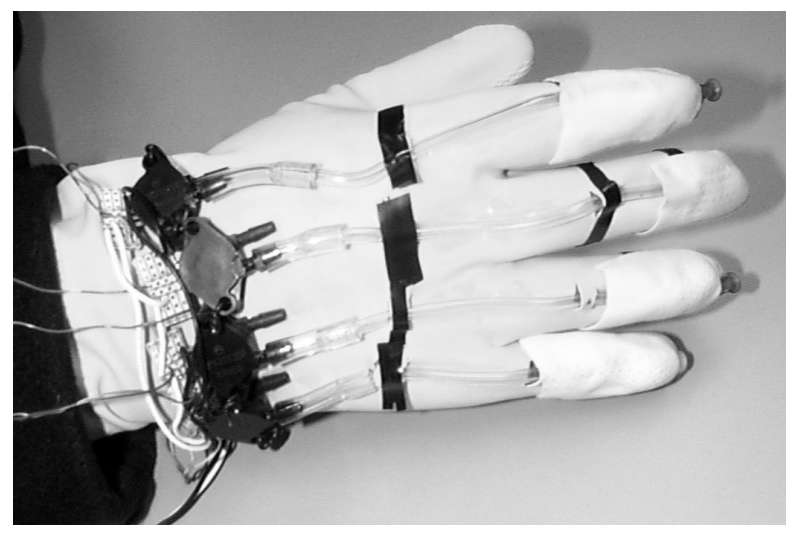

Figure 3 DataGlove Prototype

\section{4: Accuracy of measure}

For the seven people tested the fastest frequency to completely open and close the hand is $\mathbf{1 6}$ times per second, or $\boldsymbol{8 H} \boldsymbol{z}$, with an average frequency of $\boldsymbol{9}$ times per second or $4.5 \mathrm{~Hz}$. At our sampling rate of $240 \mathrm{~Hz}$, this corresponds to a worst case of one sample every $12^{\circ}$. At the comfortable hand movement speed of $2.6 \mathbf{H z}$, sampling will occur at least every $\mathbf{4}^{\circ}$. At any speed the measured accuracy range had an error of $2.5^{\circ}$ to $3^{\circ}$.
In order to test out the Glove, a series of measurements were taken with the gloved hand grabbing onto cylindrical object of a known diameter. The results are plotted in Figure 4. Due to the "one size fits" approach taken in our prototype a certain amount of slack, and thus error, was introduced into our measure. As a result diameters of less then a half-centimeter were indistinguishable from a closed fist, while diameters greater then 9.5 centimeters became indistinguishable from a completely open hand. Over the operational range of one to nine centimeters of diameter, Figure 4 confirms that the glove does indeed provide the linear relationship between angle and pressure predicted by Equation 2 .

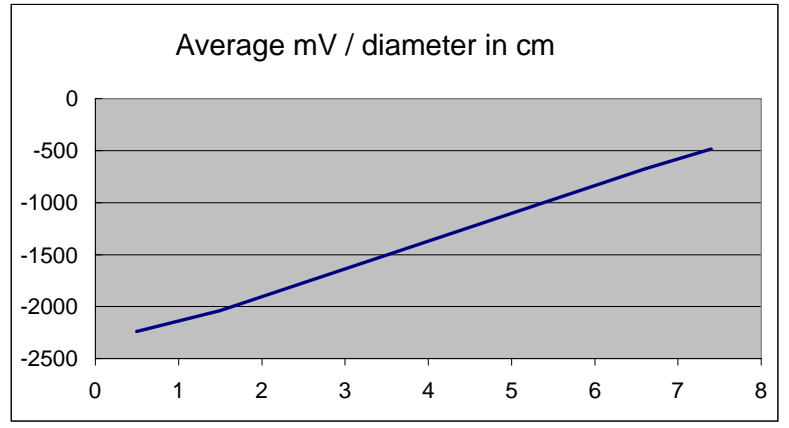

\section{Figure 4 Data Glove finger physical overview}

\section{5: Conclusion}

We have shown how inexpensive pressure sensors can be used to measure joint bending. While we demonstrated this in a dataglove, the technique can sense one degree of freedom in joint motion for any joint in the body. While these results are in themselves significant enough to call the prototype a success, it is worth noting that these are the accuracy measurements for the crudely constructed prototype. It is not hard to imagine that any commercially produced glove would greatly reduce the sources of error and increase accuracy significantly.

\section{6: Acknowledgements}

Thanks to William Dietze for technical help and equipment, and to Danyel Fisher, Mark Billinghurst, and David Starke for editing assistance.

\section{7: References}

[1] A Generic P/N Precision Temperature Sensor http://www.national.com/pf/LM/LM235.html

[2] Reference on pressure sensor technology

http://mot-sps.com/sps/General/chips-nav.html 